\title{
Survival During Treatment Period of Patients with Severe Heart Failure Admitted to Intensive Care Unit (ICU) at Gondar University Hospital (GUH), Gondar, Ethiopia
}

\author{
Azmera Hailay $^{1,}$, Essey Kebede ${ }^{2}$, Kasim Mohammed ${ }^{3}$ \\ ${ }^{1}$ Department of Statistics, College of Natural \& Computational Sciences, Mekele University, Mekele, Ethiopia \\ ${ }^{2}$ Department of Statistics, College of Natural \& Computational Sciences, Bahir-Dar University, Bahir-Dar, Ethiopia \\ ${ }^{3}$ Department of Statistics, College of Natural \& Computational Sciences, University of Gondar, Gondar, Ethiopia \\ Email address: \\ azmerahailay@gmail.com (A. Hailay), kebedeessey@yahoo.com (E. Kebede), kasim.fti@gmail.com (K. Mohammed)
}

\section{To cite this article:}

Azmera Hailay, Essey Kebede, Kasim Mohammed. Survival During Treatment Period of Patients with Severe Heart Failure Admitted to Intensive Care Unit (ICU) at Gondar University Hospital (GUH), Gondar, Ethiopia. American Journal of Health Research.

Vol. 3, No. 5, 2015, pp. 257-269. doi: 10.11648/j.ajhr.20150305.11

\begin{abstract}
Heart failure (HF) is progressive and irreversible which occurs more slowly because of damage to the heart muscle, building up through time due to disease of the heart or a blood vessel leading from the heart as a result of various diseases. In nearly all regions of the world HF is both common and increasing. Predictions for the next two decades include tripling of heart disease and stroke mortality in Latin America, the Middle East, and even sub-Saharan Africa. This study is an attempt to study the survival during treatment period of severe heart failure patients admitted to intensive care unit(ICU) ward at Gondar University Hospital(GUH). Data were collected from 147 severe heart failure patients admitted to the ICU ward of Gondar University Hospital, Gondar, Ethiopia during January 2012- June 2014. Non-parametric, semi-parametric PH, parametric PH and AFT models were used for data analysis. Different statistical techniques were used to compare performances of semi-parametric PH, parametric PH and AFT models. Descriptive statistical results show that predominant causes of HF were coronary heart disease and valvular heart disease which causes $38.1 \%$ and $29.9 \%$ of the total population respectively. In this study $(26.09 \%)$ of the deaths were attributed to respiratory failure, $(15.22 \%)$ due to cardiac arrest (15.22\%) multi-organ failure, (10.87\%) end stage renal failure. The variables "History of HF", "Duration of HF", and "Department the patient seen at first" were found to be significant predictors of the survival during treatment period of patients with severe heart failure admitted to ICU ward by the multivariable Cox PH model and the multivariable exponential and Weibull PH, and multivariate Log-Logistic AFT models. The Cox PH model was a better fit than the other models. Being seen at chronic illness follow-up first, diagnosed as patient with heart failure for the first time and lower number of years stay with heart failure significantly decrease hazard of in-ICU mortality. Special attention to patients discharged from emergency department, diagnosed as patient with heart failure before current admission, with higher number of years stay with heart failure, advanced age and with comorbidity condition coronary artery disease is recommended as possible interventions to improve in-ICU survival of patients with severe HF.
\end{abstract}

Keywords: Intensive Care Unit(ICU), Heart Failure(HF), Survival Analysis, Cox Proportional Hazards(PH) Model, Parametric Proportional Hazards model, Accelerated Failure Time (AFT) Model

\section{Introduction}

Heart failure is often used to mean chronic heart failure or congestive heart failure (CHF). Clinically it is impossible to define HF by a single term as a result it is defined as a complex clinical syndrome in which there is a functional or structural impairment in the heart. This can result from any functional or structural cardiac disorder and it impairs the ventricle's ability to fill with or eject blood and to deliver oxygenated blood corresponding to the requirements of the metabolizing tissues of the body and/or doing so at increased filling pressures (Agval, 2014).

Heart failure (HF) is progressive and irreversible which occurs more slowly because of damage to the heart muscle, building up through time due to disease of the heart or a blood vessel leading from the heart as a result of various 
diseases, accordingly it is a serious clinical condition which represents the end-stage of numerous other cardiac diseases (Ponikowski; et al., 2014).

Heart failure is a major clinical problem worldwide, reaching an epidemic level in the developed world with no known cure at this time. Approximately 26 million people worldwide are living with heart failure, and nearly 1 million new cases are diagnosed annually worldwide, making it the most rapidly growing cardiovascular disorder. In economically developed countries, up to one person in five is expected to develop heart failure at some point in their life (Ponikowski; et al., 2014)andit affects1-3\% of the general population (Owusu and Boakye, 2013; McMurray and Stewart, 2002). It is predominantly seen in the geriatric population in these countries, with almost $80 \%$ of cases occurring in patients over the age of 65 . Thus, prevalence of heart failure has been shown to follow an exponential pattern, which rises with age and affects $6-10 \%$ of people over age 65 (Owusu and Boakye, 2013).

Despite improvements in care over the past 20 years, the outlook for patients with heart failure remains poor, and it has a higher mortality than many of the common malignancies (Agval, 2014; Ponikowski; et al., 2014). One year mortality in developed countries is approximately $20 \%$ while the 5-year mortality is approximately 50-65\% in population-based studies (Agval, 2014). Across the globe, $17-45 \%$ of patients admitted to hospital with heart failure die within 1 year of admission and the majority die within 5 years of admission. Besides survival rates are better for those treated in outpatient clinics, which typically have less severe symptoms than those treated in hospital (Ponikowski; et al., 2014). About $2-17 \%$ of individuals admitted to hospital with heart failure die while in hospital (Mayosi, 2007).

In a study conducted in Ghana the high prevalence of heart failure of $76 \%$ seen in the study supports the fact that HF is a major contributor to cardiovascular disease burden in subSaharan Africa. Similar findings have been reported from Cameroon where heart failure is found to be the fifth to sixth cause of hospital admissions. In other parts of sub-Saharan Africa, heart failure has been found to account to $5 \%$ to $10 \%$ of hospital admissions (Owusu and Boakye, 2013).

Compared to studies from other parts of the world, heart failure in Africa tends to occur at a much younger age with most cases recorded around the 5th and 6th decade and it is not a disease of the elderly in sub-Saharan Africa (Ponikowski; et al., 2014).This young age reflect the major contribution of rheumatic valvular disease to heart failure, but could also be accounted for infections as it remain a common cause of heart failure in many parts of the world including Africa and can strike at any age. Hospital case fatality among those with heart failure in Africa ranges from $9 \%$ to $12.5 \%$. This consistent death rate ranks heart failure among the major causes of death of cardiovascular origin in Africa (Cabral; et al., 2011; Kengne; et al., 2008).

In Ethiopia, Misganaw; et al. in their systematic review, aimed to document the prevalence and mortality associated with major non-communicable diseases in Ethiopia found cardiovascular disease accounted for 3\%-12.6\% hospital admission and found to have increased between 1970s and 2000s. This systematic review found congestive heart failure reported to have caused $2.5 \%$ of deaths among all age-groups in a sampled hospital-based mortality study (Misganaw; et al., 2014). Similarly a study conducted by analyzing surveillance data on causes of death in Addis Ababa found that, the leading cause of death was cardiovascular disease causing $24 \%$ of all death. Congestive heart failure is found to be the third cause of death following hypertension and stroke among the cardiovascular disease deaths (Misganaw; et al., 2012).

\subsection{Statement of the Problem}

In nearly all regions of the world HF is both common and increasing andit is predicted that the number of patients with $\mathrm{HF}$ to increase in countries with ageing populations. In the USA, there were 5.8 million patients living with heart failure in 2012, and this is expected to rise to 8.5 million by 2030 (Cook; et al.,2013).

Nowadays cardiovascular disease has become one of the major causes of premature death and disability in low- and middle-income countries. Moreover, predictions for the next two decades include tripling of heart disease and stroke mortality in Latin America, the Middle East, and even subSaharan Africa, a rate of increase that exceeds than for any other region, except for Asian and Pacific Island countries (ICDS, 2004). However, heart failure as cardiovascular complication remains unexplored largely in Africa (Bennett;et al., 2012).

The long-term prognosis associated with HF is also poor (Cook; et al.,2013). Reis et al. found that the three-year mortality from CHF related to severity of symptoms and ranges from $40 \%$ in patients in NYHA functional class I to $82 \%$ in those in class IV (Reis; et al., 1997). A Hospital-based study conducted in Italy also found the highest mortality rates in the ICU and emergency department with 19 and $78 \%$ deaths, respectively (Grigioni; et al., 2002).

But little research work has been reported on HF patients admitted to Intensive Care Units (ICUs), clinical department in which only critically ill patients with NYHA class IV were treated, worldwide. Furthermore, all the population based follow-up studies in survival of HF patients are based on the semi-parametric proportional hazards model.To fill these gap this thesis is aimed to investigate the survival during treatment period of severe heart failure patients admitted to intensive care unitward of Gondar University Hospital using the Cox Proportional Hazards model, Parametric Proportional Hazards model and Accelerated Failure Time models.

\subsection{Specific Objectives}

- To model survival during treatment period of patients with severe HF admitted to ICU ward

- To study the influence of predictors in-ICU ward survival of patients with severe HF

- To examine etiologies of heart failure of patients with 
severe $\mathrm{HF}$ admitted to ICU ward

- To investigate cause of death of patients with severe heart failure

- To compare Cox PH, parametric PH and AFT models in explaining the data set

\subsection{Significance of the Study}

The findings from this research are hoped to be useful in providing information about the risk factors or the most influential covariates that have significant impact on survival during treatment period of patients with severe heart failure during ICU ward admission at Gondar University Hospital and to identify death risk extent of patients under these significant factors.

\section{Materials\& Methods}

\subsection{Data Collection}

The data used in this study is obtained from Gondar University Hospital, north western Ethiopia, in Gondar city Amhara regional state of Ethiopia. The data for this study was obtained specifically from the intensive care unit (ICU) ward of the hospital, there were 147 heart failure patients admitted to the ward, all of whom were in NYHA class IV. All patients admitted to ICU ward within the indicated twenty nine months in the hospital had information on their chart on demographic profiles, clinical variables, etiology, comorbidity and other clinical variables at admission to the ward maintained by the ICU unit.

\subsection{Variables Included in the Study}

\subsubsection{Dependent Variable}

Survival analysis always measures the time from a defined starting point to the occurrence of a given event. In this study the response variable measures the length of treatment time in days from ICU ward entry to event or censoring and the event of interest is death.

\subsubsection{Independent Variables}

Table 1. Description and coding of categorical predictor variables, GUH, 2014.

\begin{tabular}{ll}
\hline Variable Name & Variable description \\
\hline Agecat & Age of the patient \\
Sex & Sex of the patient \\
Residence & Residence of the patient \\
MS & Marital status of the patient \\
HFpatient & History of heart failure \\
Dptseenf & Department seen first by the patient \\
Causeofhf & Etiology of heart failure \\
DM & Presence of Diabetes Mellitus \\
HTN & Presence of Hypertension \\
CKD & Presence of Chronic Kidney Disease \\
Stroke & Presence of Stroke \\
HIV & Presence of HIV \\
Pneumonia & Presence of Pneumonia \\
CAD & Presence of Coronary Artery Disease \\
TB & Presence of Tuberculosis \\
\hline
\end{tabular}

The most important expected correlates of the survival experience of patients with HF from literature reviews and their theoretical justification included in this study are grouped as clinical and demographic variables, and comorbidity conditions. Categorical predictors included in this study are given below in table-1. Continuous predictors are systolic blood pressure, weight, heart rate, white blood cell count (WBCC) and duration of heart failure.

\subsection{Survival Analysis}

\subsubsection{Survivor Function and Hazard Function}

The survival and hazard functions are key concepts in survival analysis for describing the distribution of survival times. The survivor functionS(t); is the probability that the survival time of a randomly selected subject is greater than some specified time $t$ or the probability of an individual being event-free beyond time $t$. In order to find the survival function, suppose $T$ be random variable associated with the survival times, $t$ be the observed value of the random variable $T$ and $f(t)$ be the underlying probability density function of the survival time $t$. The cumulative distribution function $F(t)$ represents the probability that an individual selected at random will have a survival time less than or equal to the specified value $t$. Thus, the cumulative distribution function and the survivor function are given by:

$$
\begin{aligned}
& \mathrm{F}(\mathrm{t})=\mathrm{P}(\mathrm{T} \leq \mathrm{t})=\int_{0}^{t} f(u) d u, \mathrm{t} \geq 0 \\
& S(t)=P(T>t)=1-F(t), t \geq 0
\end{aligned}
$$

The relationship between $S(t)$ and $f(t)$ is given as

$$
f(t)=\frac{d}{d t} F(t)=\frac{d}{d t}(1-S(t))=-\frac{d}{d t} S(t), t \geq 0
$$

Hazard function $\mathrm{h}(\mathrm{t})$

The hazard function is generally denoted by $h(t)$ and can be used to express the risk orhazard of death at time $t$.It is obtained from the probability that an individual dies in an infinitesimally small interval $(\mathrm{t}, \Delta t)$ given that the individual has survived up to time $t$ i.e. $P\{t \leq T<\Delta t \mid T \geq t\}$.

There is a clearly defined relationship between $S(t)$ and $h(t)$ which is given by the formula

$$
\begin{gathered}
h(t)=\frac{\mathrm{f}(\mathrm{t})}{1-\mathrm{F}(\mathrm{t})}=\frac{f(t)}{\mathrm{S}(\mathrm{t})}=\frac{-\mathrm{d}}{\mathrm{dt}} \ln \mathrm{S}(\mathrm{t}) \\
\mathrm{S}(\mathrm{t})=\exp \left[-\int_{0}^{t} h(u) d u\right]=\exp (-H(t)), t \geq 0
\end{gathered}
$$

Where $\mathrm{H}(\mathrm{t})=\int_{0}^{t} h(u) d u$ the cumulative hazard function, which can be obtained from

$$
\mathrm{H}(\mathrm{t})=-\log (\mathrm{S}(\mathrm{t}))
$$

The probability density function of $T$ can be written as

$$
f(t)=h(t) S(t)
$$




\subsubsection{Non-Parametric Methods}

(i) Kaplan-Meier Estimator of the Survival Function

Suppose $t_{1}, t_{2}, \ldots, t_{n}$ be the survival times ofn independent observations and $t_{1} \leq t_{2} \leq \cdots t_{m}, \mathrm{~m} \leq \mathrm{n}$ be the $m$ distinct ordered death times. Then the Kaplan-Meier estimator of thesurvivorship function (or survival probability) at time $t$, $\mathrm{S}(\mathrm{t})=\mathrm{P}(\mathrm{T}>t)$ is defined as:

$$
\hat{s}(t)=\prod_{t_{j} \leq \mathrm{t}} \frac{\mathrm{n}_{j}-d_{\mathrm{j}}}{\mathrm{n}_{\mathrm{j}}}=\prod_{t_{\mathrm{j}} \leq \mathrm{t}}\left[1-\frac{\mathrm{d}_{\mathrm{j}}}{\mathrm{n}_{\mathrm{j}}}\right]
$$

Where, $\mathrm{n}_{j}$ is the number of individuals who are at risk of dying at time $t_{j}$, and $d_{j}$ is the number of individuals who failed (died) at timet $t_{j}$. The variance of the $\mathrm{KM}$ survival estimator which is also known as the Greenwood's formula is given by

$$
\operatorname{var}(\hat{s}(t))=(\hat{s}(\mathrm{t}))^{2} \sum \frac{d_{\mathrm{j}}}{n_{j}\left(n_{\mathrm{j}}-d_{j}\right)}
$$

(ii) Comparison of Survivorship Functions

The simplest way of comparing the survival times obtained from two or more groups is to plot the Kaplan-Meier curves for these groups on the same graph. However, this graph does not allow us to say whether or not there is a real difference between the groups. Assessing whether or not there is a real difference between groups can only be done by utilizing statistical tests. Thus, the Mantel- Hanzel (1959), currently called the "log-rank" test is used for comparison of two or more survival distributions in this thesis work.

Let $t_{1} \leq t_{2} \leq \cdots t_{m}$ be the $\mathbf{m}$ distinct ordered death times across two groups. Suppose that $d_{j}$ failures occur at $t_{(j)}$ and that $n_{j}$ subjects are at risk just prior to $t_{(j)}(\mathrm{j}=1,2, \ldots, \mathrm{m})$. Let $d_{i j}$ and $n_{i j}$ be the corresponding numbers in group $\mathrm{i}(\mathrm{i}=1,2)$. Then the log-rank test compares the observed number of deaths with the expected number of deaths for group $i$. Consider the null hypothesis: $S(1)=S(2)$; i.e. there is no difference between survival curves in two groups. Given $\mathrm{n}_{j}$ and $d_{j}$ the random variable $\mathrm{d}_{1 \mathrm{j}}$ has the hypergeometric distribution

$$
\frac{\left(\begin{array}{c}
d_{j} \\
d_{1 j}
\end{array}\right)\left(\begin{array}{c}
n_{j}-d_{j} \\
n_{1 j}-d_{1 j}
\end{array}\right)}{\left(\begin{array}{c}
n_{j} \\
n_{1 j}
\end{array}\right)}
$$

Under the null hypothesis, the probability of experiencing an event at $t_{(j)}$ does not depend on the group, i.e. the probability of experiencing an event at $t_{j}$ is $\frac{d_{j}}{n_{j}}$. So that the expected number of deaths in group one is

$$
E\left(d_{1 j}\right)=e_{1 j}=\frac{n_{1 j} d_{j}}{n_{j}}
$$

The test statistic is given by the difference between the total observed and expected number of deaths in group one

$$
U_{L}=\sum_{j=1}^{m}\left(d_{1 j}-e_{1 j}\right)
$$

Since $d_{1 j}$ has the hypergeometric distribution, the variance of $d_{1 j}$ is given by

$$
v_{1 j}=\operatorname{Var}\left(d_{1 j}\right)=\frac{n_{1 j} n_{2 j} d_{j\left(n_{j}-d_{j}\right)}}{n_{j}^{2}\left(n_{j}-1\right)}
$$

So that the variance of $U_{L}$ is given by

$$
\operatorname{Var}\left(U_{L}\right)=\sum_{j=1}^{m} v_{1 j}=V_{L}
$$

Under the null hypothesis, statistic has an approximate normal distribution with zero mean and variance $V_{L}$. This then follows $\frac{U_{L}{ }^{2}}{V_{L}} \sim x^{2}{ }_{1}$

The general form of the test statistic to test the equality of survival curves which can also be used by several alternatives to the log-rank test, such as the Wilcoxon test, may be defined as follows:

$$
Q=\frac{\sum_{j=1}^{m} w_{j}\left(d_{i j}-\hat{e}_{1 j}\right)}{\sum_{j=1}^{m} w_{j}^{2} \hat{v}_{1 j}}
$$

Where: $w_{j}$ are weights whose values depend on the specific test

Log rank test is based on weights equal to one, i.e. $w_{j}=1$. And it is appropriate when hazard functions for two groups are proportional over time, i.e. $h_{1}(t)=\psi h_{2}(t)$.

\subsubsection{Regression Models for Survival Data}

The use of a statistical model improves on these methods by allowing survival to be assessed with respect to several factors simultaneously, and in addition, offers estimates of the strength of effect for each constituent factor. Therefore, statistical models such as Cox proportional hazards model, parametric proportional hazards model and accelerated failure time models are important and frequently used tools which, when constructed appropriately, offer valuable insight into the survival process.

(i) Cox Proportional Hazards Regression Model

Cox (1972) proposed a semi-parametric hazards model for the survival data to see the effect of explanatory variables on the hazard function. In Cox proportional hazards model, the hazard of an individual consists of the product of two terms namely the baseline hazard function $h_{0}(t)$ i.e.the hazard function when all covariates equal to zero and the exponential function of the subject's variables weighted by the regression coefficients. The hazard for the $i^{\text {th }}$ individualwith the set of covariates $x_{i}=\left(x_{1}, x_{2}, \ldots, x_{p}\right)^{\prime}$ is defined as

$$
h_{i}(\mathrm{t})=\mathrm{h}_{0}(\mathrm{t}) \exp \left(\beta_{1} \mathrm{x}_{1 \mathrm{i}}+\beta_{2} \mathrm{x}_{2 \mathrm{i}}+\cdots+\beta_{\mathrm{p}} \mathrm{x}_{\mathrm{pi}}\right)=\mathrm{h}_{0}(t) e^{\boldsymbol{\beta}^{\prime} x_{i}}
$$

Where, $\beta^{\prime}=\beta_{1}, \beta_{2}, \ldots, \beta_{p}$ is a column vector of $p$ regression coefficients 
The above model is called as semi-parametric model for the reason that $\mathrm{h}_{0}(t)$ is an arbitrary function meaning no assumption is made about its actual form.

With time-fixed covariates $x_{i}$, Cox regression model assumes that the hazards of any two individuals are proportional over time i.e. the ratio of the hazards is the same at any time.The hazard ratio (HR) or relative hazard for two individuals, one with covariate values $x_{i}$ and the other with all covariate values zero is defined below.

$$
\frac{h_{i}(\mathrm{t})}{\mathrm{h}_{0}(t)}=e^{\boldsymbol{\beta}^{\prime} x_{i}}
$$

(ii) Estimation of Cox Regression Model

The $\beta$ coefficients in the proportional hazards model can be estimated by maximum likelihood estimation method. Which attempts to maximize the likelihood function for the observed data simultaneously with respect to $h_{0}(t)$ and $\beta$ s.A more popular approach is proposed by Cox (1972) in which a partial likelihood function that does not depend on $h_{0}(t)$ is obtained for $\beta$ in the presence of nuisance parameterh $h_{0}(t)$.

Let $t_{(1)}<t_{(2)}<\cdots<t_{(r)}$ are ordered death times, so that $t_{(j)}$ is the $\mathrm{j}^{\text {th }}$ ordered death time. Then $R\left(\mathrm{t}_{(\mathrm{j})}\right)$ denotes the set of individuals who are at risk at time $t_{(j)}$. So that $R\left(\mathrm{t}_{(\mathrm{j})}\right)$ is the group of individuals who are alive and uncensored at a time just prior to $t_{(j)}$ and it is called the risk set.

Probability of one death at time $t_{(j)}$ is the sum of the probabilities of death at time $t_{(j)}$ over all individuals who are at risk of death at that time. If these individuals are indexed by $l$ then the partial likelihood function is given by

$$
\mathrm{L}_{\mathrm{P}}(\beta)=\prod_{\mathrm{j}=1}^{\mathrm{r}} \frac{\exp \left(\beta^{\prime} x_{j}\right)}{\sum_{l \in R\left(t_{j}\right)} \exp \left(\beta^{\prime} x_{l}\right)}
$$

The log partial likelihood function is

$$
\operatorname{LL}_{\mathrm{P}}(\beta)=\sum_{\mathrm{j}=1}^{r}\left[\beta^{\prime} x_{j}-\ln \left(\sum_{l \in R\left(t_{j}\right)} \exp \left(\beta^{\prime} x_{l}\right)\right)\right]
$$

The estimates of $\beta$ parameters can be found by using Newton-Raphson procedure.

The partial likelihood derived above is valid when there are no ties in the data set. The simplest approximation to the likelihood function proposed by Kalbfleisch and Prentice to be used in the presence of tied observations is given by Breslow and Peto. This method is usually the default procedure for handling ties in statistical software for survival analysis.

Let $S_{j}$ be the vector of sums of each of the p covariates for those individuals who die at the $\mathrm{j}^{\text {th }}$ death time, $t_{j}, j=$ $1,2, \ldots, r$. If there are $d_{j}$ deaths at $t_{j}$, the $\mathrm{h}^{\text {th }}$ elements of $S_{j}$ is $S_{h j}=\sum_{k=1}^{d_{j}} x_{h j k}$, where $x_{h j k}$ is the value of the $\mathrm{h}^{\text {th }}$ explanatory variable, $h=1,2, \ldots, p$ for the $k^{\text {th }}$ of $d_{j}$ individuals, $k=1,2, \ldots, d_{j}$, who die at the $j^{\text {th }}$ death time. Then the Breslow's approximate likelihood is given by

$$
\prod_{j=1}^{\mathrm{r}} \frac{\exp \left(\beta^{\prime} S_{j}\right)}{\left\{\sum_{l \in R\left(t_{j}\right)} \exp \left(\beta^{\prime} x_{l}\right)\right\}^{d_{j}}}
$$

\subsubsection{Parametric Proportional Hazards Model}

(i) Weibull Proportional Hazards model

Conceptually Weibull proportional hazards model including Exponential PH model as a special case when the shape parameter $\gamma$ is unity, is similar to $\mathrm{Cox} \mathrm{PH}$ model. The hazard ratio is interpreted similarly in both models. The effect of predictors in the Weibull PH model is also multiplicative on hazard scale.

The baseline hazard function for Weibull $\mathrm{PH}$ model with scale parameter $\lambda$ and shape parameter $\gamma$ is:

$$
h_{0}(t)=\lambda \gamma(t)^{\gamma-1}
$$

Under the Weibull PH model, the hazard function of a particular $i^{\text {th }}$ individual with covariates $\left(x_{1}, x_{2}, \ldots, x_{p}\right)$ is given by

$$
\begin{gathered}
h_{i}(t)=h_{0}(t) \exp \left(\beta_{1} \mathrm{x}_{1 \mathrm{i}}+\beta_{2} \mathrm{x}_{2 \mathrm{i}}+\cdots+\beta_{\mathrm{p}} \mathrm{x}_{\mathrm{pi}}\right) \\
=\lambda \gamma(t)^{\gamma-1} e^{\boldsymbol{\beta}^{\prime} \boldsymbol{x}_{i}}
\end{gathered}
$$

Where $\beta_{1}, \beta_{2}, \ldots, \beta_{\mathrm{p}}$ areunknown regression coefficients

The cumulative hazard function of the WeibullPHmodel for the $i^{t h}$ individual is given by:

$$
H_{i}(t)=\exp \left(\beta^{\prime} x_{i}\right) \lambda t^{\gamma}
$$

The corresponding survival function is given by

$$
S_{i}(t)=\exp \left\{-\exp \left(\beta^{\prime} x_{i}\right) \lambda t^{\gamma}\right\}
$$

The WeibullPH model is estimated by maximum likelihood estimation method. The likelihood function of $n$ observations maximized with respect to unknown parameters $\beta_{1}, \beta_{2}, \ldots, \beta_{p}$, and $\lambda$ and $\gamma$. The likelihood function of any $\mathrm{PH}$ model is given by:

$$
L=\prod_{i=1}^{n}\left\{\frac{f\left(t_{i}\right)}{S\left(t_{i}\right)}\right\}^{\delta_{i}} S_{i}\left(t_{i}\right)
$$

(ii) Accelerated Failure Time Model

AFT models work to measure the effect of covariate to "accelerate" or to "decelerate" survival time meaning the effect of covariate is multiplicative on time scale. Under AFT models the survival function of the $i^{\text {th }}$ individual with covariates $\left(x_{1}, x_{2}, \ldots, x_{p}\right)$ at time $t$ is the same as the survival function of an individual with a baseline survival function at a time $t / e_{e^{\beta^{\prime} x_{i}}}$, mathematically it can be expressed with its corresponding hazard function as:

$$
S_{i}(t)=S_{0}\left[t / e^{\beta^{\prime} x_{i}}\right], h_{i}(t)=e^{-\beta^{\prime} x_{i}} h_{0}\left(t / e^{\beta^{\prime} x_{i}}\right)
$$

Where, $\beta^{\prime}=\left(\beta_{1}, \beta_{2}, \ldots, \beta_{p}\right)$ is a vector of regression coefficients, $S_{0}($.$) and h_{0}($.$) are the baseline survival and$ hazard functions respectively. The effect size for the AFT 
model is measured using the time ratio (TR), which is a ratio of the survival time of an individual with an exposure to the survival time of an individual without the exposure for a given survival probability.

Suppose $T_{i}$ is a random variable representing the survival time for the $i^{\text {th }}$ individual. Then the general log-linear form of the AFT model shows the mathematical relation between the log of time and the set of covariates expressed as follows:

$$
\log T_{i}=\mu+\beta^{\prime} x_{i}+\delta \varepsilon_{i}
$$

Where, $\beta^{\prime}=\left(\beta_{1}, \beta_{2}, \ldots, \beta_{p}\right), \mu$ is intercept, $\delta$ is scale parameter and $\varepsilon_{i}$ is a random variable used to model the deviation of values of $\log T_{i}$ from the linear part of the model. $\varepsilon_{i}$ is assumed to have a particular probability distribution supposed to be followed by the survival time under study.

AFT models are fitted using the maximum likelihood estimation method. The likelihood function of $\mathrm{n}$ observed survival times, $t_{1}, t_{2}, \ldots, t_{n}$ for the log-linear form of the AFT model is given by

$$
L(\beta, \mu, \sigma)=\prod_{i=1}^{n}\left[f_{i}\left(t_{i}\right)\right]^{\delta_{i}}\left[S_{i}\left(t_{i}\right)\right]^{\left(1-\delta_{i}\right)}
$$

Where $f_{i}\left(t_{i}\right)$ and $S_{i}\left(t_{i}\right)$ are the density and survival functions for the $i^{\text {th }}$ individual at time $t_{i}$ and $\delta_{i}$ is the event indicator for the observation and has value zero for censored and one for uncensored individuals. If $f_{\varepsilon_{i}}\left(z_{i}\right)$ and $S_{\varepsilon_{i}}\left(z_{i}\right)$ are probability density function and survival function respectively of the random variable $\varepsilon_{i}$ in such a way that

$$
S_{i}\left(t_{i}\right)=S_{\varepsilon_{i}}\left(z_{i}\right)
$$

and

$$
f_{i}\left(t_{i}\right)=\frac{1}{\sigma t_{i}} f_{\varepsilon_{i}}\left(z_{i}\right)
$$

Where,

$$
z_{i}=\left(\frac{\log t_{i}-\left(\mu+\beta_{1} x_{1 i}+\beta_{2} x_{2 i}+\cdots+\beta_{P} x_{P i}\right)}{\sigma}\right)
$$

\subsection{Comparison of Cox PH and Parametric Models}

Different models can be compared on the basis of the variables selected and their coefficients in each model, goodness of fit tests such as $\mathrm{R}^{2}$ type statistic and Cox-Snell residuals plot.

\subsection{Comparison of Model Estimate}

If the models being compared have a similar set of covariates that have entered in the respective final models, it can be interpreted as all models are equally good or bad as far as theidentification of important covariates associated with the outcome. However, it is difficult to interpret either way if the selected variables in the models being compared are different, as there is no way of knowing the truth (Khanal, 2009).
The precision of the regression coefficients is another criterion that can be used to compare different models. The smaller the standard error, the more precise an estimate is expected to be. A model with more precise coefficients can be considered as a more precise model.

But as discussed earlier, Cox regression model, Exponential and Weibull $\mathrm{PH}$ models work on $\mathrm{PH}$ metric whereas Log-Logistic and Log-normal AFT models work only on AFT metric. Because of this, regression coefficients of a Cox model, Exponential or Weibull PH model cannot be directly compared with those of Log-Logistic and Lognormal AFT models. However regression coefficients of Cox $\mathrm{PH}$, Exponential $\mathrm{PH}$ and Weibull $\mathrm{PH}$ can be compared directly, as all works on PH metric and similarly all AFT models are directly comparable for the precision of theestimates, as all AFTs work on AFT metric (Khanal, 2009).

\subsection{Comparison of $R^{2}$ Type Statistic}

$\mathrm{R}^{2}$ Type statistic is a relative measure for the improvement in log-likelihood value from the model without any covariate to the final model. Hence two or more relative measures can be compared irrespective of the type of log-likelihood estimated. The model with more $\mathrm{R}^{2}$ type is indicative of a better model than a model with a low $\mathrm{R}^{2}$.

\subsection{Comparison Based on Cox-Snell Residuals Plots}

The construction of the Cox-Snell residuals plot is explained in the respective sections above. Broadly, all models require the plot to be a straight line, passing through the origin to qualify for a good fit. So the plots under each model can be visually assessed as to which one of them is close to the requirements of a good fit.

\section{Results}

\subsection{Descriptive Summaries and Non-Parametric Analysis}

The study was based on 147 patients, which is obtained from Gondar university hospital ICU ward. Among the patients considered, $31.3 \%$ (46) of them were dead while the rest $68.7 \%$ (101) were censored. The median time of a patient stay in the ICU ward to treatment until death or censoring is 6 days.

The predominant causes of HF were coronary heart disease and valvular heart disease which caused $38.1 \%$ and $29.9 \%$ of the total population respectively. Other etiologies found in this study were hypertension $8.8 \%$, anemia $6.1 \%$, atrial fibrillation $4.0 \%$ and unknown causes $12.9 \%$. For the reason that $\mathrm{HF}$ is not a disease by itself patients with HF have other causes of death. In this study deaths were attributed to respiratory failure $(26.09 \%)$, cardiac arrest $(15.22 \%)$, multiorgan failure $(15.22 \%)$, end stage renal failure $(10.87 \%)$, cardiogenic shock $(6.52 \%)$, septic shock $(4.35 \%)$, pneumonia $(4.35 \%)$ and non-defined causes $(17.39 \%)$.

The mean age of patients with severe heart failure was 48.04years for men and 50.83 years for women. The mean baseline body weight was $60.18 \mathrm{~kg}$ and $59.158 \mathrm{~kg}$ for male and female patients, respectively. The mean systolic blood pressure was 116.45 for men and 112.72 for women. The 
mean heart rate was95.74bpm and $98.24 \mathrm{bpm}$ for male and female patients respectively. The mean white blood cell count was 9.05 for men and 11.79 for women. The mean duration of heart failure was 3.90years for men and5.01years for women. Descriptive measures such as, median treatment time, number of censored and death for each categorical predictor variables included in the study are presented below in table-2.

Table 2. Results of descriptive measures of categorical predictor variables, GUH, 2014.

\begin{tabular}{|c|c|c|c|c|}
\hline \multirow{2}{*}{ Covariates } & \multirow{2}{*}{ Category } & \multirow{2}{*}{$\begin{array}{l}\text { Median treatment } \\
\text { time (n) }\end{array}$} & \multicolumn{2}{|l|}{ Status } \\
\hline & & & Censored (\%) & Death (\%) \\
\hline \multirow{3}{*}{ Age of patients } & $(0,49]$ & $6(72)$ & $52(72.2)$ & $20(27.8)$ \\
\hline & $(49,65]$ & $5.5(36)$ & $28(77.8)$ & $8(22.2)$ \\
\hline & $(65, \infty)$ & $6(39)$ & $21(53.8)$ & $18(46.2)$ \\
\hline \multirow{2}{*}{ Sex of patients } & Female & $6(92)$ & $67(72.8)$ & $25(27.2)$ \\
\hline & Male & $6(55)$ & $34(61.8)$ & $21(38.2)$ \\
\hline \multirow{2}{*}{ Residence of patients } & Urban & $6(90)$ & $57(63.3)$ & $33(77.2)$ \\
\hline & Rural & $6(57)$ & $44(36.7)$ & $13(22.8)$ \\
\hline \multirow{3}{*}{ Marital status of patients } & Single & $6(28)$ & $22(78.6)$ & $6(21.4)$ \\
\hline & Married & $6(96)$ & $63(65.6)$ & $33(34.4)$ \\
\hline & Divorced/Widowed & $7(23)$ & $16(69.6)$ & $7(30.4)$ \\
\hline \multirow{2}{*}{ History of heart failure } & New & $6(38)$ & $23(60.5)$ & $15(39.5)$ \\
\hline & HF patient before & $6(109)$ & $78(71.6)$ & $31(28.4)$ \\
\hline \multirow{3}{*}{ Department seen first } & Medical OPD & $7(86)$ & $64(74.4)$ & $22(25.6)$ \\
\hline & Emergency & $5(41)$ & $17(41.5)$ & $24(58.5)$ \\
\hline & Chronic illness follow up & $6(20)$ & $20(100)$ & 0 \\
\hline \multirow{6}{*}{ Etiology of heart failure } & CHD & $7(56)$ & $40(71.4)$ & $16(28.6)$ \\
\hline & VHD & $6(44)$ & $30(68.2)$ & $14(31.8)$ \\
\hline & Hypertension & $5(13)$ & $10(76.9)$ & $3(23.1)$ \\
\hline & Anemia & $6(9)$ & $4(44.4)$ & $5(55.6)$ \\
\hline & Atrial Fibrillation & $8.5(6)$ & $3(50.0)$ & $3(50.0)$ \\
\hline & Unknown & $6(19)$ & $14(73.7)$ & $5(26.3)$ \\
\hline \multirow{2}{*}{ Diabetes Mellitus } & Yes & $6(14)$ & $7(50.0)$ & $7(50.0)$ \\
\hline & No & $6(133)$ & $94(70.7)$ & $39(29.3)$ \\
\hline \multirow{2}{*}{ Hypertension } & Yes & $6(31)$ & $22(70.9)$ & $9(29.1)$ \\
\hline & No & $6(116)$ & $79(68.1)$ & $37(31.9)$ \\
\hline \multirow{2}{*}{ Chronic Kidney Disease } & Yes & $8(17)$ & $11(64.7)$ & $6(35.3)$ \\
\hline & No & $6(130)$ & $90(69.2)$ & $40(30.8)$ \\
\hline \multirow{2}{*}{ Stroke } & Yes & $6(4)$ & $2(50.0)$ & $2(50.0)$ \\
\hline & No & $6(143)$ & $99(69.2)$ & $44(30.8)$ \\
\hline \multirow{2}{*}{ HIV } & Yes & $8(9)$ & $7(77.8)$ & $2(22.2)$ \\
\hline & No & $6(138)$ & $94(68.1)$ & $44(31.9)$ \\
\hline \multirow{2}{*}{ Pneumonia } & Yes & $6(49)$ & $31(63.3)$ & $18(36.7)$ \\
\hline & No & $6(98)$ & $70(71.4)$ & $28(28.6)$ \\
\hline \multirow{2}{*}{ Coronary Artery Disease } & Yes & $4(11)$ & $7(63.6)$ & $4(36.4)$ \\
\hline & No & $6(136)$ & $94(69.1)$ & $42(30.9)$ \\
\hline \multirow{2}{*}{ Tuberculosis } & Yes & $5(5)$ & $3(60.0)$ & $2(40.0)$ \\
\hline & No & $6(142)$ & $98(69.0)$ & $44(31.0)$ \\
\hline
\end{tabular}

\subsection{Cox Proportional Hazards Model}

The Cox model identified significant predictors at 25\% level. Consequently, the candidate variables for building a multivariate Cox model are department the patient seen at first, place of residence, sex of the patient, history of HF, age category of the patient, presence of diabetes mellitus as comorbidity, duration of heart failure and presence of coronary artery disease as comorbidity. The hazards ratio, $95 \%$ 
confidence intervals and standard errorsfor each variable is given below in table-3.

Table 3. Results of univariate Cox analysisGUH, 2014.

\begin{tabular}{|c|c|c|c|c|}
\hline Covariates & HR & $75 \%$ CI & P_value & SE \\
\hline Sex & 0.62 & $\left(\begin{array}{l}0.35 \\
1.11)\end{array}\right.$ & 0.109 & 0.18 \\
\hline Residence & 1.75 & (0.92 3.34) & 0.086 & 0.58 \\
\hline Coronary artery disease & 2.03 & $(0.725 .72)$ & 0.182 & 1.07 \\
\hline Diabetes mellitus & 1.70 & $(0.763 .80)$ & 0.198 & 0.70 \\
\hline History of HF & 0.68 & $(0.371 .26)$ & 0.219 & 0.21 \\
\hline White blood cell count & 0.98 & (0.92 1.03) & 0.411 & 0.03 \\
\hline \multicolumn{5}{|l|}{ Department seen first } \\
\hline Medical OPD & 1 & & & \\
\hline Emergency & 3.00 & $(1.675 .36)$ & 0.000 & 0.89 \\
\hline Duration of $\mathrm{HF}$ & 1.04 & (0.99 1.09) & 0.144 & 0.03 \\
\hline Age category & & & 0.097 & \\
\hline Less than 49 years & 1 & & & \\
\hline $50-65$ years & 0.93 & $(0.412 .11)$ & 0.863 & 0.39 \\
\hline Above 65 years & 1.76 & (0.93 3.33) & 0.081 & 0.57 \\
\hline Marital status & 1.18 & $(0.393 .51)$ & 0.792 & 0.26 \\
\hline Systolic BP & 1.003 & (0.99 1.01) & 0.608 & 0.01 \\
\hline Weight & 0.98 & $\left(\begin{array}{lll}0.95 & 1.02\end{array}\right)$ & 0.361 & 0.02 \\
\hline Heart rate & 0.996 & (0.98 1.009) & 0.554 & 0.01 \\
\hline Cause of HF & 1.29 & $(0.374 .44)$ & 0.506 & 0.09 \\
\hline Hypertension & 0.96 & (0.46 1.98) & 0.906 & 0.36 \\
\hline Chronic kidney disease & 1.09 & $(0.462 .58)$ & 0.845 & 0.48 \\
\hline Stroke & 2.04 & $(0.498 .51)$ & 0.327 & 1.49 \\
\hline Tuberculosis & 2.00 & $(0.488 .31)$ & 0.342 & 1.45 \\
\hline HIV & 0.45 & $\left(\begin{array}{lll}0.11 & 1.88\end{array}\right)$ & 0.256 & 0.33 \\
\hline Pneumonia & 1.22 & $(0.682 .22)$ & 0.502 & 0.37 \\
\hline
\end{tabular}

The significant variables at $25 \%$ level were considered in a forward stepwise manner with an entry probability 0.05 and removal probability 0.251 . Among the candidate variables considered for building multivariate Cox,stepwise procedure picked up three variables, history of HF, department the patient seen at first and duration of heart failure. The HR, 95\% confidence intervals and standard error for each variable in the fitted model are shown in table-4.

Table 4. Multivariate Cox regression modelfor HF data, GUH, 2014.

\begin{tabular}{lllll}
\hline Covariates & HR & 95\% CI & P value & SE \\
\hline History of HF & 0.32 & $(0.150 .68)$ & 0.003 & 0.12 \\
Department seen first & 3.32 & $(1.855 .98)$ & 0.000 & 1.00 \\
Emergency & 1.08 & $(1.031 .14)$ & 0.003 & 0.03 \\
Duration of HF & & &
\end{tabular}

Plot of the Cox-Snell residuals was applied to test the overall fit of the model. In this method Cox-Snell residuals were plotted against the cumulative hazard of Cox-Snell residuals as shown in figure-1. The figure reveals that the overall fit of the Cox model is good. However, there is little evidence of a systematic deviation from the straight line at the right, this can be expected even if we have a well-fitting Cox model because of the reduced effective sample size caused by prior failures and censoring (Khanal, 2009). The $\mathrm{R}^{2}$ type statistic was also calculated as follows:

$$
\begin{gathered}
R_{p}{ }^{2}=1-\left\{\exp \left[\frac{2}{147}(-206.4725-(-190.75384))\right]\right\} \\
=0.192538029
\end{gathered}
$$

Which conveys $19.25 \%$ of variation in the partial loglikelihood is explained by Cox PH model.

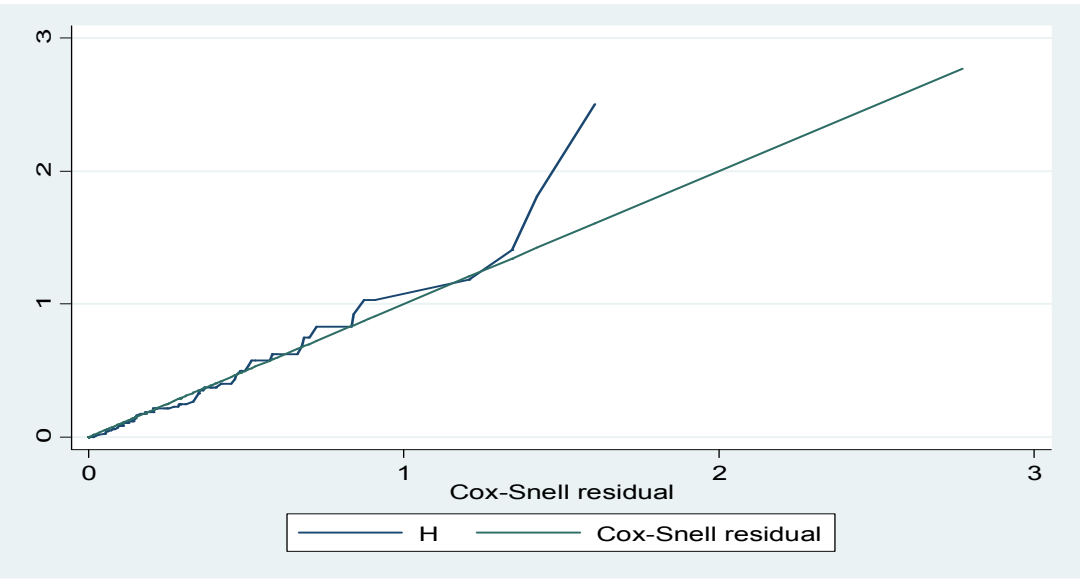

Figure 1. Cumulative hazard plot of the Cox-Snell residual for multivariate Cox PH model. 


\subsection{Parametric Proportional Hazards Model}

The results of univariateparametric $\mathrm{PH}$ models are presented in table- 5 . In both models variables significant at $25 \%$ level in the univariate analysis were taken as candidate variables for their multivariate analysis. Stepwise forward selection procedure was also implemented for these models as used in multivariate Cox model.

Table 5. Results of univariateWeibull PH model and Exponential PH, GUH, 2014.

\begin{tabular}{|c|c|c|c|c|c|c|c|c|}
\hline \multirow{2}{*}{ Covariates } & \multicolumn{4}{|c|}{ Weibull } & \multicolumn{4}{|c|}{ Exponential } \\
\hline & HR & $75 \% \mathrm{CI}$ & Pvalue & SE & HR & $75 \% \mathrm{CI}$ & P value & SE \\
\hline Sex & 0.57 & $\left(\begin{array}{lll}0.32 & 1.02\end{array}\right)$ & 0.059 & 0.17 & 0.63 & $(0.351 .13)$ & 0.121 & 0.19 \\
\hline Residence & 1.76 & $(0.933 .34)$ & 0.084 & 0.58 & 1.72 & $(0.913 .28)$ & 0.096 & 0.56 \\
\hline CAD & 2.34 & $(0.836 .62)$ & 0.109 & 1.24 & 1.71 & $(0.614 .78)$ & 0.303 & 0.90 \\
\hline Diabetes mellitus & 1.64 & $(0.733 .67)$ & 0.228 & 0.67 & 1.69 & $(0.763 .78)$ & 0.201 & 0.69 \\
\hline History of HF & 0.75 & $(0.401 .39)$ & 0.243 & 0.24 & 0.71 & $(0.381 .31)$ & 0.270 & 0.22 \\
\hline WBCC & 0.98 & $\left(\begin{array}{lll}0.93 & 1.03\end{array}\right)$ & 0.391 & 0.02 & 0.98 & (0.92 1.03) & 0.416 & 0.03 \\
\hline \multicolumn{9}{|l|}{ Department } \\
\hline Medical OPD & 1 & & & & & & & \\
\hline Emergency & 3.14 & $(1.765 .61)$ & 0.000 & 0.93 & 2.84 & (1.59 5.06) & 0.000 & 0.84 \\
\hline Duration of HF & 1.04 & (0.99 1.09) & 0.114 & 0.03 & 1.04 & (0.99 1.09) & 0.139 & 0.03 \\
\hline \multicolumn{9}{|l|}{ Age category } \\
\hline Less than $49 \mathrm{Y}$ & 1 & & & & & & & \\
\hline $50-65$ years & 1.01 & $(0.442 .29)$ & 0.985 & 0.42 & 0.92 & $\left(\begin{array}{ll}0.41 & 2.09\end{array}\right)$ & 0.843 & 0.38 \\
\hline Above $65 \mathrm{Y}$ & 1.75 & $(0.923 .30)$ & 0.086 & 0.57 & 1.75 & $(0.923 .30)$ & 0.086 & 0.56 \\
\hline Marital status & 0.99 & $\left(\begin{array}{lll}0.61 & 1.63\end{array}\right)$ & 1.00 & 0.25 & 1.08 & $(0.671 .76)$ & 0.751 & 0.27 \\
\hline Systolic BP & 1.00 & (0.99 1.02) & 0.467 & 0.01 & 1.00 & (0.99 1.01) & 0.676 & 0.01 \\
\hline Weight & 0.98 & $(0.951 .02)$ & 0.398 & 0.02 & 0.99 & $\left(\begin{array}{lll}0.95 & 1.02\end{array}\right)$ & 0.443 & 0.02 \\
\hline Heart rate & 0.99 & (0.98 1.01) & 0.408 & 0.01 & 0.99 & $(0.981 .01)$ & 0.586 & 0.01 \\
\hline Cause of HF & 1.06 & $(0.911 .25)$ & 0.445 & 0.09 & 1.05 & $\left(\begin{array}{lll}0.90 & 1.24\end{array}\right)$ & 0.531 & 0.08 \\
\hline Hypertension & 0.85 & $(0.411 .77)$ & 0.676 & 0.32 & 0.90 & $(0.43$ 1.87) & 0.779 & 0.33 \\
\hline CKD & 1.15 & $(0.492 .71)$ & 0.753 & 0.50 & 1.14 & $(0.482 .68)$ & 0.770 & 0.50 \\
\hline HIV & 0.44 & $(0.111 .81)$ & 0.256 & 0.32 & 0.51 & $(0.122 .11)$ & 0.355 & 0.37 \\
\hline Pneumonia & 1.25 & $(0.692 .25)$ & 0.465 & 0.38 & 1.24 & $\left(\begin{array}{lll}0.69 & 2.25)\end{array}\right.$ & 0.469 & 0.38 \\
\hline
\end{tabular}

Both final multivariate exponential and Weibull PH models picked up the same three variables namely history of HF, duration of HF and department the patient seen at first as selected by multivariate Cox PH model. The hazard ratio and corresponding 95\% CI with standard error for both models are given in table-6. More or less both models had same HR with almost identical standard errors in estimating the variables history of HF and duration of HF, while in estimating the variable department the patient seen at first exponential achievedconsiderably smaller standard error.

Table 6. Results of multivariate Weibull PH modeland Exponential PH model, GUH, 2014.

\begin{tabular}{|c|c|c|c|c|c|c|c|c|}
\hline \multirow{2}{*}{ Covariates } & \multicolumn{4}{|c|}{ Weibull } & \multicolumn{4}{|c|}{ Exponential } \\
\hline & HR & $95 \%$ CI & Pvalue & SE & HR & $95 \%$ CI & P value & SE \\
\hline History of HF & 0.33 & $\left(\begin{array}{lll}0.15 & 0.72)\end{array}\right.$ & 0.006 & 0.13 & 0.37 & $(0.170 .79)$ & 0.011 & 0.14 \\
\hline Duration of HF & 1.09 & (1.03 1.16) & 0.002 & 0.03 & 1.08 & (1.02 1.13) & 0.006 & 0.03 \\
\hline $\begin{array}{l}\text { Department seen first } \\
\text { Emergency }\end{array}$ & 4.71 & $(2.608 .51)$ & 0.000 & 1.42 & 3.82 & $(2.146 .82)$ & 0.000 & 1.13 \\
\hline
\end{tabular}

The overall goodness of fit of both models was also assessed using the Cox-Snell residuals plot (Figure-2). 

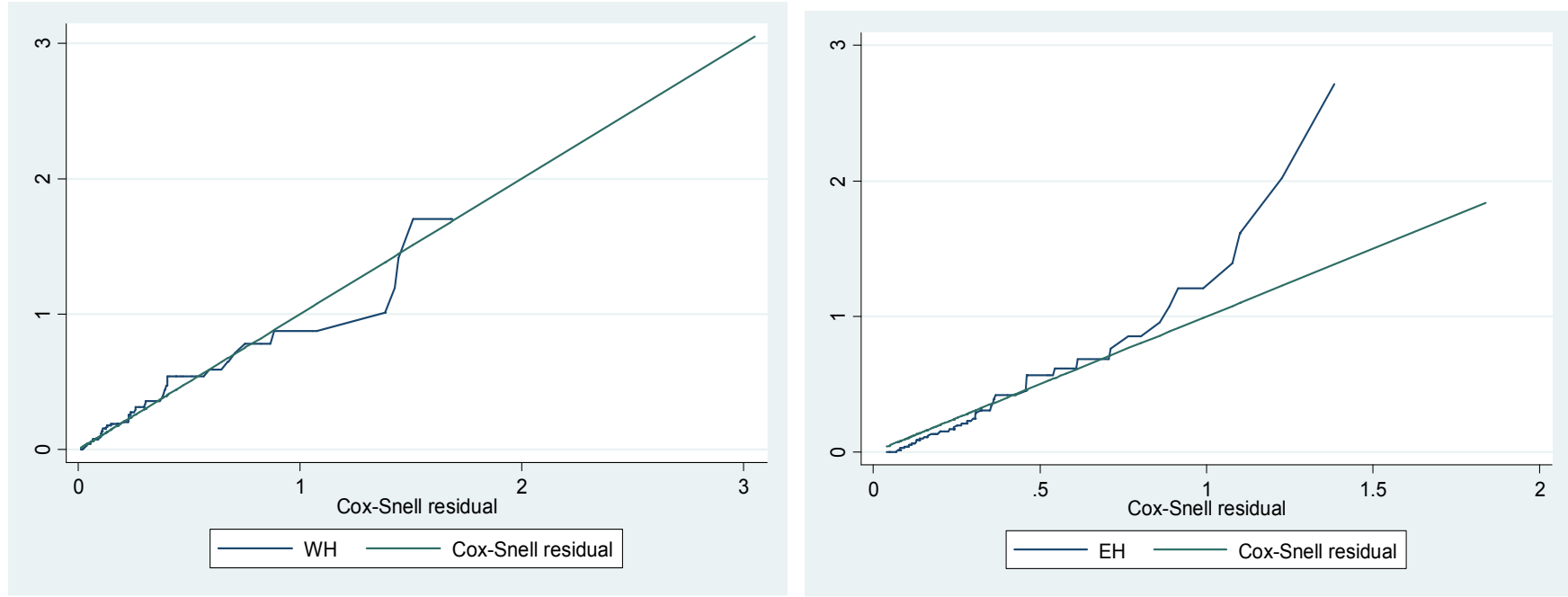

whereWH is for Weibull PH model and EH for Exponential PH model

Figure 2. Cox-Snell residualsplot ofWeibull PH model and exponential PH model, GUH, 2014.

Cox-Snell residuals plot for Weibull PH model shows deviation from the straight line passing through origin, almost at residuals size 1 while the exponential shows deviation at size greater than 1 as shown in figure-2.

$\mathrm{R}^{2}$ type statistic was also obtained for both models in the same way as obtained in Cox PH model. $\mathrm{R}^{2}$ type statistic for Exponential PH model is calculated as follows:

$$
R_{p}^{2}=1-\left\{\exp \left[\frac{2}{147}(-105.1385-(-90.26696))\right]\right\}=
$$

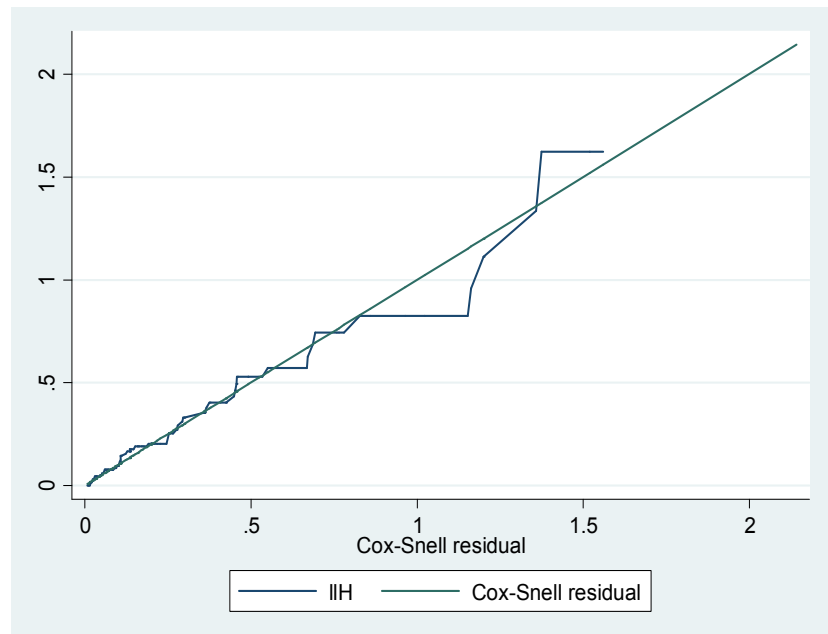

Which conveys $18.32 \%$ variation in the full log-likelihood is explained by the fitted exponential model. By the same fashion $\mathrm{R}^{2}$ type is found to be 0.1626 for Weibull which indicated that $16.26 \%$ variation in the full log-likelihood is explained by the fitted Weibull model. Results of $\mathrm{R}^{2}$ type statistic and Cox-Snell residuals plot may indicate that the overall fit of the Weibull PH model is less satisfactory than exponential.

\subsection{AFT Models}

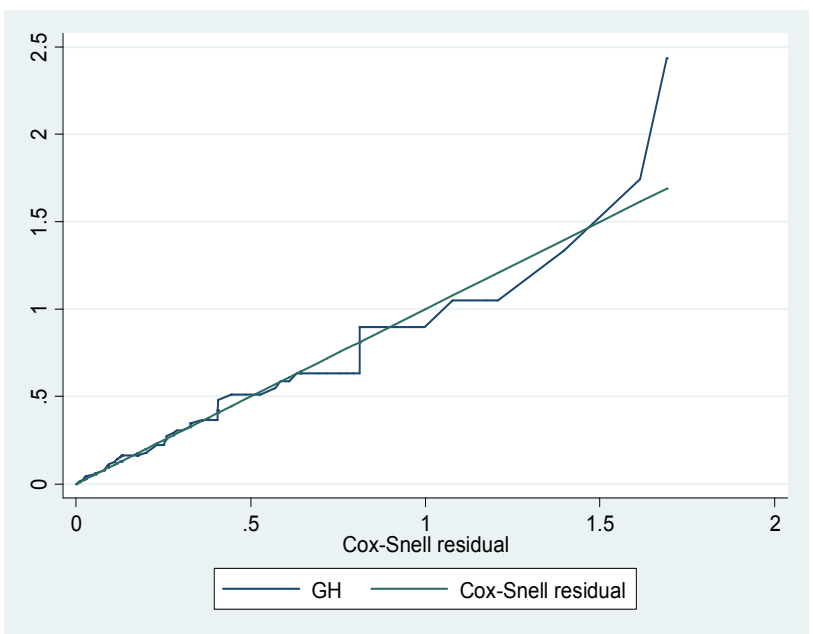

$11 \mathrm{H}$ for Log-Logistic and GH for Generalised gamma

Figure 3. Cox-Snell residuals plot of Log-LogisticVs Generalized-Gamma, GUH, 2014.

Table 7. Results of multivariate Log-Logistic AFT model, GUH, 2014.

\begin{tabular}{lllll}
\hline Covariates & TR & $\mathbf{9 5 \%}$ CI & P value & SE \\
\hline History of HF & 1.73 & $(1.132 .65)$ & 0.012 & 0.38 \\
Department emergency & 0.45 & $(0.320 .63)$ & 0.000 & 0.08 \\
Duration & 0.96 & $(0.930 .99)$ & 0.014 & 0.01 \\
\hline
\end{tabular}


Table 8. Results of multivariate Log-normal and Generalized-Gamma AFT models, GUH, 2014.

\begin{tabular}{lllllllll}
\hline Log-normal & \multicolumn{7}{c}{ Gamma } \\
\hline Covariates & TR & $95 \%$ CI & P value & SE & TR & $95 \%$ CI & P value & SE \\
History of HF & 1.49 & $(1.052 .11)$ & 0.025 & 0.26 & 1.50 & $(1.062 .12)$ & 0.021 & 0.26 \\
Department Emergency & 0.44 & $(0.320 .62)$ & 0.000 & 0.07 & 0.44 & $(0.320 .62)$ & 0.000 & 0.07 \\
CAD & 0.54 & $(0.310 .96)$ & 0.034 & 0.16 & 0.52 & $(0.290 .92)$ & 0.024 & 0.15 \\
Age category Above 65Y & 0.64 & $(0.460 .90)$ & 0.011 & 0.11 & 0.63 & $(0.450 .89)$ & 0.008 & 0.11 \\
\hline
\end{tabular}

The Cox-Snell residuals plot of generalized-gamma deviates from straight line at residuals size of almost 0.6 while the plot for Log-Logistic deviates at size almost 1 . $\mathrm{R}^{2}$ type statistic was calculated for both Log-Logistic and generalized-gamma models. The $\mathrm{R}^{2}$ type for Log- Logistic was calculated as follows:

$$
\begin{gathered}
R_{p}^{2}=1-\left\{\exp \left[\frac{2}{147}(-104.3941-(-90.0074))\right]\right\} \\
=0.17777188
\end{gathered}
$$

In the same way $\mathrm{R}^{2}$ type is found to be 0.167414353 for generalized gamma. Results of $\mathrm{R}^{2}$ type convey $16.74 \%$ variation in full log-likelihood was explained by the fitted generalized- gamma model and $17.78 \%$ of variability in full log-likelihood is explained by the Log-Logistic AFT model. The $\mathrm{R}^{2}$ type statistic and Cox-Snell residuals plot may be indicators of Log-Logistic model as better performed model than generalized-gamma model.

The Q-Q plot of the variable with two levels in the fitted AFT models, history of HF, is used to check the adequacy of AFT model. The Q-Q plot in Figure-4 approximates to a straight line indicating that the AFT model may provide an appropriate fit for survival time data. Thus, HF data might be modeled suitably by the AFT models.

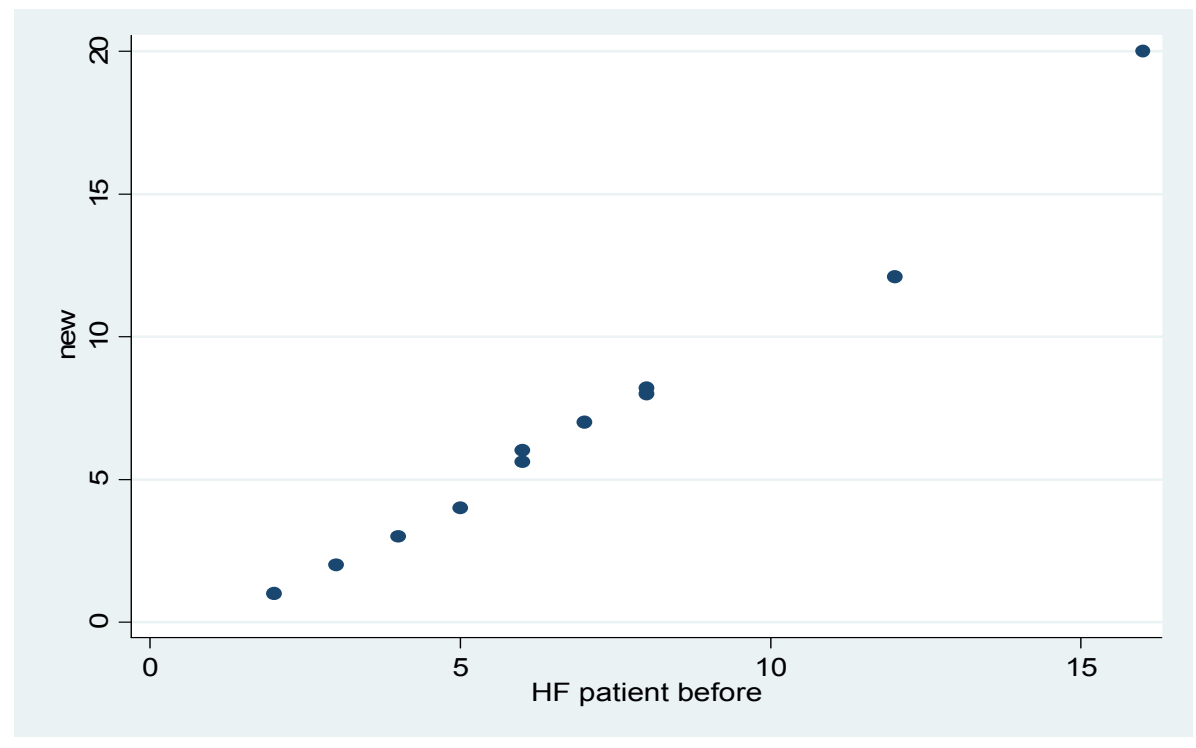

Figure 4. Q-Q plot of history of HF, GUH, 2014.

\subsection{Comparison of Models}

The hazard ratio and corresponding 95\% CI with standard error for each variable of both models together are shown in table-9. Both models are the same in estimating duration of HF with identical HR and SE. Hazard ratios of the two variables, history of HF and department the patient seen at first, for exponential PH model are more than those of Cox model with corresponding larger standard errors comparative with Cox. Therefore, Cox results are more precise than exponential results. Based on the explained variation, 19.25\% of variation in the partial log-likelihood is explained by the Cox $\mathrm{PH}$ model which is greater than that of the variation explained in the full log-likelihoodby exponential model (18.32\%). Thus, Cox proportional hazards model is better performed than exponential Proportional hazards model.

Table 9. Comparison of model estimates: Cox PH model vs. exponential PH model, GUH, 2014.

\begin{tabular}{|c|c|c|c|c|c|c|c|c|}
\hline \multirow{2}{*}{ Covariates } & \multicolumn{4}{|l|}{$\operatorname{Cox}$} & \multicolumn{4}{|c|}{ Exponential } \\
\hline & HR & $95 \%$ CI & P value & SE & HR & $95 \% \mathrm{CI}$ & P value & SE \\
\hline History of HF & 0.32 & $(0.150 .68)$ & 0.003 & 0.12 & 0.37 & $\left(\begin{array}{lll}0.17 & 0.79)\end{array}\right.$ & 0.011 & 0.14 \\
\hline Duration of HF & 1.08 & (1.03 1.14) & 0.003 & 0.03 & 1.08 & (1.02 1.13) & 0.006 & 0.03 \\
\hline Department seen first Emergency & 3.32 & $(1.855 .98)$ & 0.000 & 1.00 & 3.82 & $(2.146 .82)$ & 0.000 & 1.13 \\
\hline
\end{tabular}




\subsection{Discussion of Results}

The proportionality of hazards assumption was satisfied accordingly exponential model is taken in its $\mathrm{PH}$ metric in order to ease comparison with Cox PH. Cox PH model estimates were more precise than estimates of the exponential PH model. Explained variation and Cox-Snell residuals plot also shows Cox PH fitted to data better than exponential distribution this is in line with reports choose Cox over parametric models (Khanal, 2009; Conge and Tsoikos, 2010).

History of HF and emergency department were significant factors which affected the survival probability of patients in multivariate analysis for all considered models. Based on Cox multivariate analysis newly diagnosed patients as patient with heart failure at current admission had an estimated $68 \%$ lower risk of mortality than patients diagnosed as patients with heart failure before current admission $(\mathrm{HR}=0.32)$. This finding was similar to a study which pointed a lower risk of in-Hospital mortality if diagnosed as HF patient for the first time (Abraham; et al.,2009). Similarly based on Cox multivariate analysis, it is estimated that patients first seen at emergency department experiences 3.32 times higher risk of mortality than patients first seen at medical OPD $(\mathrm{HR}=3.32)$. This finding was similar to study which pointed discharge of patients directly from the emergency department as associated with an increased risk of hospital mortality (Grimaldi; et al., 2014).

In multivariate generalized-gamma AFT analysis age was found to be a strong predictor variable which revealedpatients agebove 65 years were at increased risk of in-ICU mortality than patients aged less than 49 years $(\mathrm{TR}=0.63)$, and our finding was similar with previous reports (Abraham; et al., 2009; Grigioni; et al., 2002; Gustafsson; et $a l ., 2003$ ) indicating advanced age associated with higher inhospital mortality and (Capell; et al., 2013; Pocock; et al., 2006) agree inpatients aged above 65 were at lower survival experience. Multivariate generalized-gamma AFT model was also discovered patients with presence of coronary artery disease as comorbidity were at increased risk of in-ICU mortality than patients without coronary artery disease.

Furthermore, the non-parametric Kaplan-Meier survival estimator curve showed female patients had slightly higher survival probability than male patients this is in line with (Barlera;et al., 2012; Capell;et al., 2013; Henkel;et al., 2008; Pocock;et al., 2006; Ho;et al., 1993) revealing female sex associated with higher survival experience in population based follow-up studies and (Gustafsson;et al., 2003) indicating male patients at higher risk of in-hospital mortality. Patients with diabetes mellitus as comorbidity had lower survival experience than diabetes mellitus absent patients based on the Kaplan-Meier survival estimator curve. our result is in line with other studies (Barlera;et al., 2012; Capell;et al., 2013; Henkel;et al., 2008; Pocock;et al., 2006; Ho;et al., 1993) revealing diabetes mellitus absent patients to have higher survival experience in population based followup studies and (Gustafsson;et al., 2003) indicating patients with diabetes mellitus as comorbidity were at higher risk of in-hospital mortality.

It is believed that when a suitable distribution can be found the parametric model is more informative than the Coxmodel. Besides, the appropriate use of these models offers the advantage of being slightly more efficient; they yield more precise estimates (Bradburn;et al., 2003; Moghimi;et al., 2008).

\section{Conclusions}

In this study, Exponential model was significant and better fitted the data than other AFT models. The adequacy of AFT assumption was checked using QQ plot and found to be straight line with slop one indicating adequacy of AFT distribution. It was found that semi-parametric Cox proportional hazards model was better fitted the data than parametric exponential proportional hazards model and AFT models. The proportionality of hazards assumption was found satisfied.

From the multivariable Cox regression model and exponential regression model it was found that the variables patient first seen at emergency department, history of HF and duration of HF were the significant correlates of survival during treatment period of patients with severe HF admitted to ICU ward at Gondar university hospital. Other models and the non-parametric Kaplan-Meier survival estimate curves found that age, sex, coronary artery disease and diabetes mellitus to have effect on survival of the patients in the ward.

The predominant causes of HF were coronary heart disease and valvular heart disease which causes $38.1 \%$ and $29.9 \%$ of the total population respectively. Deaths were attributed torespiratory failure $(26.09 \%)$, cardiac arrest $(15.22 \%)$, multi-organ failure $(15.22 \%)$, end stagerenal failure $(10.87 \%)$, cardiogenic shock (6.52\%), septic shock (4.35\%), pneumonia (4.35\%) and non-defined causes (17.39\%). Male patients and patients with diabetes mellitus were at higher risk of mortality than female patients and patients without diabetes mellitus respectively. Thus, attention should be given to male patients and patients with diabetes mellitus as comorbidity in the ward.

\section{Acknowledgements}

The authors of this article would like to the thank Gondar University Hospital (GUH) for making available the data used in this research. We also thank University of Gondar for the use of their computers and internate service.

\section{References}

[1] Abraham,W.T., Fonarow,G.C., Albert, N.M., Stough,W.G., Gheorghiade, M., Greenberg,B.H., O'Connor,C.M., Sun,J.L., Yancy,C.W., Young, J.B. (2009). Predictors of In-Hospital Mortality in Patients Hospitalized for Heart Failure. J Am CollCardiol 52:347-56

[2] Agvall, B. (2014). Heart failure in primary care with special emphasis on costs and benefits of a disease management program, Sweden PhD dissertation, Linköping University 
[3] Barlera, S., Tavazzi, L., Franzosi,M.G., Marchioli, R., Raimondi, E., Masson, S., Urso, R., Lucci, D., Nicolosi,G.L., Maggioni,A.P., Tognoni, G. (2012). Predictors of Mortality in 6975 Patients with Chronic Heart Failure in the GISSI-HF Trial: Proposal for a Nomogram. Circ Heart Fail 110-135; available from http://circheartfailure.ahajournals.org/

[4] Bennett, D.A., Eliasz,T.K., Forbes, A., Kiszely, A., Khosla, R., Petrinic, T., Praveen, D., Shrivastava, R., Xin, D., Patel, A., MacMahon, S., Rahimi, K. (2012). Study protocol: systematic review of the burden of heart failure in low- and middleincome countries. Bennett et al. Systematic Reviews 1:59

[5] Bradburn,M.J., Clark,T.G., Love,S.B., Altman,D.G. (2003). Survival Analysis Part II: Multivariate data analysis - an introduction to concepts and methods. British Journal of Cancer 89: 605-611.

[6] Bradburn,M.J., Clark,T.G., Love,S.B., Altman,D.G. (2003). Survival Analysis Part III: Multivariate data analysis choosing a model and assessing its adequacy and fit. British Journal of Cancer 89: $605-611$

[7] Cabral,T.T., Samuel,A.J., Alessandro, G., Sylvia, C., Alessandro, F., Gianfranco, B. (2011). Occurrence, etiology and challenges in the management of congestive heart failure in sub-Saharan Africa: experience of the Cardiac Centre in Shisong, Cameroon. Pan African Medical Journal 8:11

[8] Capell,E.F., Colet, J.C., Miralles, J.D., Saladich,I.J., Wensing, M., Rotellar,J.M. (2013). Survival in Mediterranean Ambulatory Patients With Chronic Heart Failure. A Population-based Study. Rev EspCardiol 66(7):539-544

[9] Cook, C., Cole, G., Asaria, P., Jabbour, R., Darrel, P. (2013). The annual global economic burden of heart failure. Int $\mathrm{J}$ Cardiol: available from: http://dx.doi.org/10.1016/j.ijcard.2013.12.028

[10] Grigioni, F., Carinci, V., Favero, L., Reggiani, L., Magnania, G., Potena, L., Barbieri, A., Magelli, C., Branzi, A., Magnani, B. (2002). Hospitalization for congestive heart failure: is it still a cardiology business? European Journal of Heart Failure 4: $99-104$

[11] Grimaldi, A., Ammirati, E., Karam, N., Vermi, A., DeConcilio, A., Trucco, G., Aloi, F., Arioli, F., Figini, F., Ferrarello, S., Maria, S. F., Grottola, R., D’Arbela, P., Alfieri, O., Marijon, E., Freers, J., Mirabel, M. (2014). Cardiac surgery for patients with heart failure due to structural heart disease in Uganda: access to surgery and outcomes. Cardiovasc J Afr 25

[12] Gustafsson, F., Torp-Pedersen, C., Brendorp, B., Seibæk, M., Burchardt, H., Køber, L. (2003). Long-term survival in patients hospitalized with congestive heart failure: relation to preserved and reduced left ventricular systolic function. European Heart Journal 24: 863-870

[13] Henkel,D.M., Redfield, M.M., Weston, S.A., Gerber, Y., Roger,V.L. (2008). Death in Heart Failure: A Community
Perspective. Circulation 1:91-97

[14] Ho, K.K., Anderson,K.M., Kannel,W.B., Grossman, W., Levy, D.(1993). Survival after the onset of congestive heart failure in Framingham Heart Study subjects. Circulation 88: 107-115

[15] International Cardiovascular Disease Statistics. (2004). Statistical Fact Sheet. American Heart Association Web Site, www.americanheart.org.

[16] Kengne,A.P., Dzudie, A., Sobngwi, E. (2008). Heart failure in sub-Saharan Africa: A literature review with emphasis on individuals with diabetes. Vascular Health and Risk Management 4(1): 123-130

[17] Khanal,S.P. (2009). Comparison of Cox proportional hazard, parametric proportional hazard and accelerated failure time models in the survival of acute liver failure. New Delhi, $\mathrm{PhD}$ dissertation, AIIMS

[18] Mayosi,B.M. (2007). Contemporary trends in the epidemiology and management of cardiomyopathy and pericarditis in sub-Saharan Africa. Heart 93:1176-83

[19] McMurray,J.J., and Stewart, S. (2002). The burden of heart failure. Eur Heart J Supplements 4 (Suppl D): D50-D58

[20] Misganaw, A., Haile-Mariam, D., Ali, A. and Araya, T. (2014). Epidemiology of Major Non-communicable Diseases in Ethiopia: A Systematic Review. J Health PopulNutr 32(1):113

[21] Misganaw, A., Haile-Mariam, D., Ali, A. and Araya, T. (2012). The Double Mortality Burden among Adults in Addis Ababa, Ethiopia, 2006-2009. Prev Chronic Dis 9:110-142

[22] Moghimi-Dehkordi, B., Safaee, A., Pourhoseingholi, M.A., Fatemi, R., Tabeie, Z., Zali,M.R. (2008). Statistical comparison of survival models for analysis of cancer data. Asian Pacific J Cancer Prev 9: 417-20

[23] Owusu,I.K., Boakye,Y.A. (2013). Prevalence and etiology of Heart Failure in Patients Seen at a Teaching Hospital in Ghana. J Cardiovasc Dis Diagn 1: 131

[24] Pocock,S.J., Wang, D., Pfeffer, M.A., Yusuf, S., McMurray,J.J., Swedberg,K.B., Stergren,J.O., Michelson,E.L., Pieper,K.S., Granger,C.B. (2006). Predictors of mortality and morbidity in patients with chronic heart failure. European Heart Journal 27: $65-75$

[25] Ponikowski, P., Anker, S.D., AlHabib,K.F., Cowie,M.R., Force,T.L., Hu, S. (2014). Heart failure: Preventing disease and death worldwide. European Society of Cardiology; Available from: http://spo.escardio.org/eslides/view.aspx?eevtid=59\&f

[26] Reis, S., Holubkov, R., Edmundowicz, D., Mcnamare, D., Zell,K.A., Detre,K.M., and Feldman, A.M. (1997). Treatment of Patients Admitted to the Hospital With Congestive Heart Failure: Specialty-Related Disparities in Practice Patterns and Outcomes. J Am CollCardiol 30:733-8 